\title{
Evidence of fixed capacity in visual object categorization
}

\author{
Alec Scharff • John Palmer • Cathleen M. Moore
}

Published online: 3 May 2011

(C) Psychonomic Society, Inc. 2011

\begin{abstract}
How is visual object perception limited by divided attention? Whereas some theories have proposed that it is not limited at all (unlimited capacity), others have proposed that divided attention introduces restrictive capacity limitations or serial processing (fixed capacity). We addressed this question using a task in which observers searched for instances of particular object categories, such as a moose or squirrel. We applied an extended simultaneous-sequential paradigm to test the fixed-capacity and unlimited-capacity models (Experiment 1). The results were consistent with fixed capacity and rejected unlimited capacity. We ascertained that these results were due to attention, and not to sensory interactions such as crowding, by repeating the experiment using a cuing paradigm with physically identical displays (Experiment 2). The results from both experiments were consistent with theories of object perception that have fixed capacity, and they rejected theories with unlimited capacity. Both serial and parallel models with fixed capacity remain viable alternatives.
\end{abstract}

Keywords Visual search · Divided attention · Object recognition · Capacity limitations · Simultaneous-sequential paradigm

Visual perception allows us to categorize objects, distinguishing a house from a train or a person from a dog. In navigating cluttered real-world environments, we are presented with multiple objects of interest simultaneously. In this article, we

\footnotetext{
A. Scharff $(\bowtie) \cdot$ J. Palmer

Department of Psychology, University of Washington,

Seattle, WA 98195, USA

e-mail: scharff@uw.edu

C. M. Moore

Department of Psychology, University of Iowa,

Iowa City, IA, USA
}

will examine how such divided attention affects our ability to categorize objects.

\section{Capacity}

The effect of divided attention can be characterized in terms of capacity. Following Broadbent (1958), capacity refers to the quantity of information that can pass through a system during a given time interval. The two extreme, boundary-defining models are unlimited-capacity and fixed-capacity models.

In unlimited-capacity models, divided attention does not limit information processing. In this case, performance is limited only by the quality of the sensory data. Importantly, unlimited-capacity processing is not necessarily fast or accurate: The definitive property is that the speed and accuracy of individual stimulus processes are not degraded by divided attention. The prototype of this class of model is the standard parallel model (Gardner, 1973; Townsend, 1974), in which multiple stimuli are analyzed in parallel and the processing rate for each object is independent of the number of objects being analyzed. Such models have been successful in predicting performance for tasks that rely on the perception of simple visual features, such as luminance, orientation, and size (see, e.g., Bonnel, Stein, \& Bertucci, 1992; Davis, Shikano, Peterson, \& Michel, 2003; Palmer, Verghese, \& Pavel, 2000; Scharff, Palmer, \& Moore, 2011). Simple-feature tasks can be made difficult and slow by adjusting such stimulus parameters as target-distractor similarity, but still they have unlimited capacity (Huang \& Pashler, 2005).

In fixed-capacity models, there is an inflexible limit on the overall rate of information processing that persists as attention is divided over multiple stimuli. An intuitive example of fixed capacity is the standard serial model, in which one process must be completed before another can begin (see, e.g., Davis et al., 2003; Townsend, 1974). Fixed-capacity models need not be serial, however; fixed capacity can also be imple- 
mented via parallel models such as Shaw's (1980) sample size model. In that model, the observer collects "samples" of information from multiple objects simultaneously. However, the sampling rate is constant and must be distributed among the objects. With more objects, each one is sampled less often, resulting in slower, less accurate decisions. Fixed-capacity models have been successful in predicting performance in word reading tasks, consistent with the subjective impression of being able to read just one word at a time (Starr \& Rayner, 2001; Scharff et al., in press).

The term intermediate capacity describes models between the aforementioned extremes. Examples include limited-resource models (Norman \& Bobrow, 1975) and models with crosstalk between the processing of different stimuli (Mozer, 1991). The more common term limited capacity refers to both intermediate and fixed capacity.

\section{Relationship to serial-parallel architecture}

Divided attention effects have also been described in terms of architecture - that is, whether multiple processes occur serially (i.e., one at a time) or in parallel (i.e., all at once). Some have argued that the categorization of objects is serial (e.g., Kahneman, Treisman, \& Gibbs, 1992; Neisser, 1967), while others have argued that it is parallel (Rousselet, Thorpe, \& Fabre-Thorpe, 2004a). This question is challenging, in part because experimental predictions derived for a particular serial model can often be mimicked by a parallel model with limited capacity (Townsend, 1974).

In principle, capacity and architecture are orthogonal. That said, measurements of capacity can be used to rule out specific parallel or serial models. In Table 1, we illustrate the most common combinations of architecture and capacity. The rows distinguish models with different limits on capacity, and the columns distinguish parallel and serial models. Here, we have filled in only the three specific models already introduced.

\section{Previous studies of capacity in object categorization}

The question of capacity limitations in object categorization has been addressed using diverse experimental paradigms.

Table 1 Models with different combinations of capacity and architecture

\begin{tabular}{lll}
\hline & Parallel & Serial \\
\hline Fixed capacity & Sample size model & $\begin{array}{c}\text { Standard serial } \\
\text { model }\end{array}$ \\
$\begin{array}{l}\text { Intermediate capacity } \\
\text { Unlimited capacity }\end{array}$ & Standard parallel model & \\
\hline
\end{tabular}

Prior studies focused on the predictions of unlimited-capacity models. Most, though not all, previous work rejected unlimited-capacity models and suggested that object perception requires limited-capacity processes. The present study is perhaps the first to explicitly test the predictions of a fixedcapacity model for object categorization.

Evans and Treisman (2005) presented observers with a rapid serial stream of objects and found that they were impaired in identifying subsequently presented target objects ("attentional blink"). This is consistent with a limited-capacity model for categorizing objects. Potter and Fox (2009) presented observers with multiple concurrent object streams and found that observers were more likely to detect target objects when monitoring fewer streams, again rejecting unlimited-capacity models.

Several visual search studies have used line drawings or images of objects as stimuli (e.g., Biederman, Blickle, Teitelbaum, \& Klatsky, 1988). These studies found large set-size effects for visual search, usually interpreted as evidence for limited-capacity processing. Most of these studies did not consider the unintended effects of set-size manipulations on sensory and decision processes, each of which can produce large set-size effects in the absence of capacity limitations (see Palmer, 1995). Thus, these studies provide ambiguous evidence for capacity limitations.

Two visual search studies by Rousselet and colleagues that did account for sensory and decision effects yielded results that supported unlimited-capacity object categorization. In these search experiments, observers indicated whether an animal image was present among one to four natural scene images (Rousselet, Fabre-Thorpe, \& Thorpe, 2002; Rousselet, Thorpe, \& Fabre-Thorpe, 2004b). The speed-accuracy response distribution was consistent with the predictions of an unlimited-capacity model. However, the interpretation of these studies is controversial. Because the target and distractor images were dissimilar in appearance (animal images vs. nonanimal images), targets may have been distinguishable from distractors on the basis of distinct simple features. Therefore, unlimited-capacity categorization may have relied on diagnostic simple features rather than on perceptual inferences of object form and function (Evans \& Treisman, 2005). In addition, these studies used consistent stimulus-response mappings (i.e., animal images always prompted a "yes" response), which promote perceptual learning (Shiffrin \& Schneider, 1977) and may have also facilitated the use of diagnostic features. Diagnostic features may be artifacts of the particular stimulus set, or they may represent a useful strategy that

Fig. 1 Schematic of experimental conditions used in Experiment 1. Each condition begins with category cues, indicating the two relevant categories for that trial. Stimuli are then presented. After the presentation the category cues return, and the observer responds by indicating which category was present in the display 


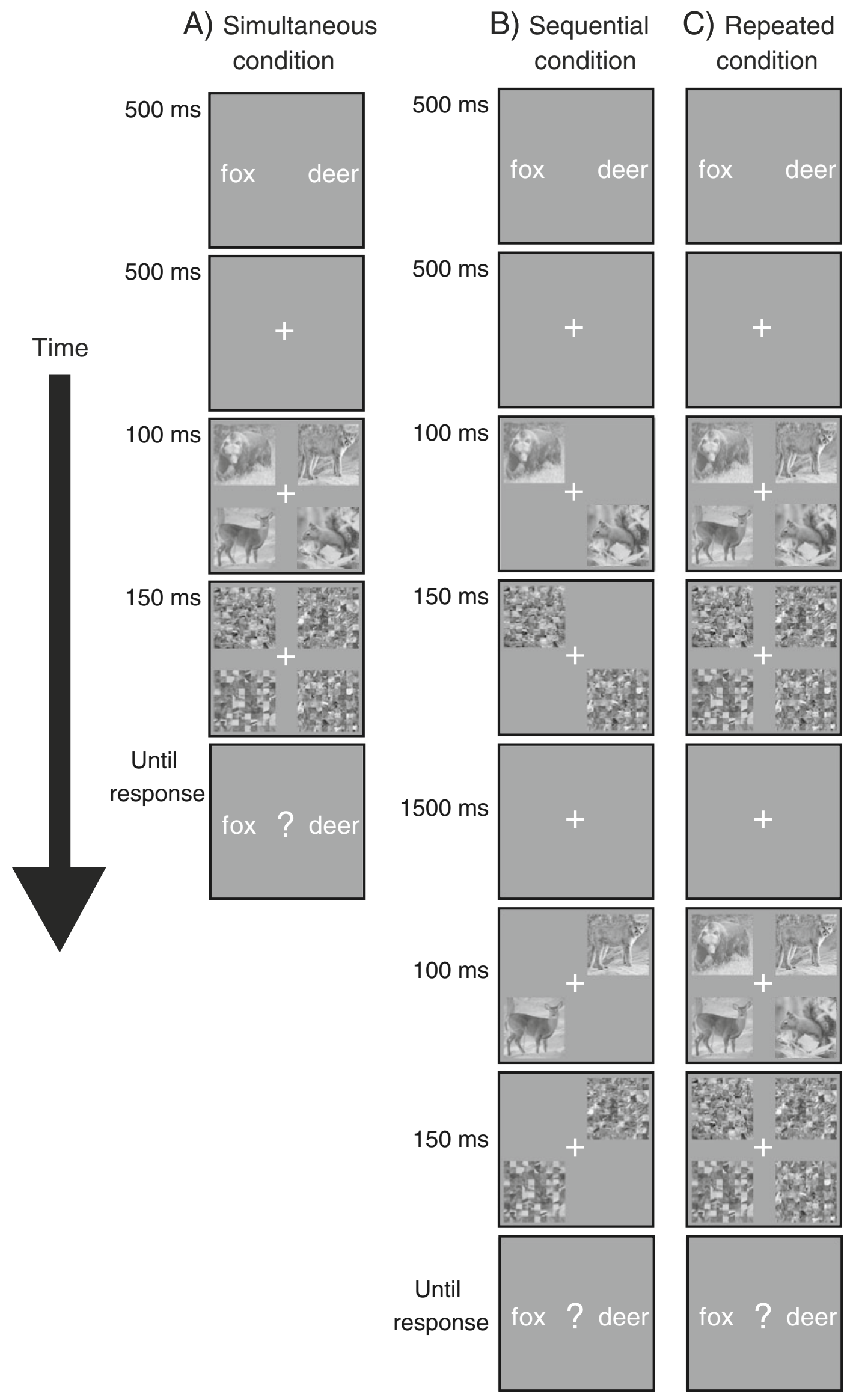


can be applied in real-world situations to avoid the "bottleneck" of object perception.

\section{The present study}

In Experiment 1, we followed a two-part strategy to evaluate capacity limitations in object categorization. First, we measured capacity using the extended simultaneoussequential paradigm, which provides predictions for both fixed-capacity and unlimited-capacity models. Second, to avoid categorization by diagnostic features, we used visually similar object categories and suppressed the learning of diagnostic features by changing stimulus-response mappings from trial to trial. The goal was to create a task in which performance depended on a full analysis of object form and function, rather than on diagnostic features.

\section{Experiment 1}

The extended simultaneous-sequential paradigm uses three conditions to distinguish capacity limitations. Each model predicts a distinct pattern of results across the conditions. The three conditions are shown schematically in Fig. 1. In all conditions, observers searched for a target drawn from one of two categories (e.g., moose or squirrel) and indicated which kind of target was present. In the simultaneous condition (column A), all four stimuli appear concurrently and persist for $100 \mathrm{~ms}$. In the sequential condition (column B), the four stimuli are divided in two subsets that appear in sequence: First, two stimuli appear in one 100-ms frame, and then the other two appear in a second 100-ms frame. In the repeated condition (column C), all four stimuli appear in each of two 100-ms frames. Critically, each presentation frame has the same display duration, $100 \mathrm{~ms}$. This presentation is followed by a $150-\mathrm{ms}$ mask in which each stimulus is replaced by a scrambled version of another image from the stimulus set. In the sequential and repeated conditions, there is a 1,250-ms blank interval between presentations, to allow time for complete attention switching (Duncan, Ward, \& Shapiro, 1994). The conditions were run in separate blocks, alternating within a session.

All models discussed thus far predict higher accuracy in the repeated condition than in the simultaneous condition. The simultaneous and repeated conditions in turn provide lower and upper bounds for accuracy in the sequential condition. As detailed below, the unlimited-capacity models predict equivalence between the simultaneous and sequential conditions, while the fixed-capacity models predict equivalence between the repeated and sequential conditions. The specific models underlying these predictions are detailed in the appendix of Scharff et al. (in press; see also Busey \& Palmer, 2008, for a discussion of how to account for task variations).

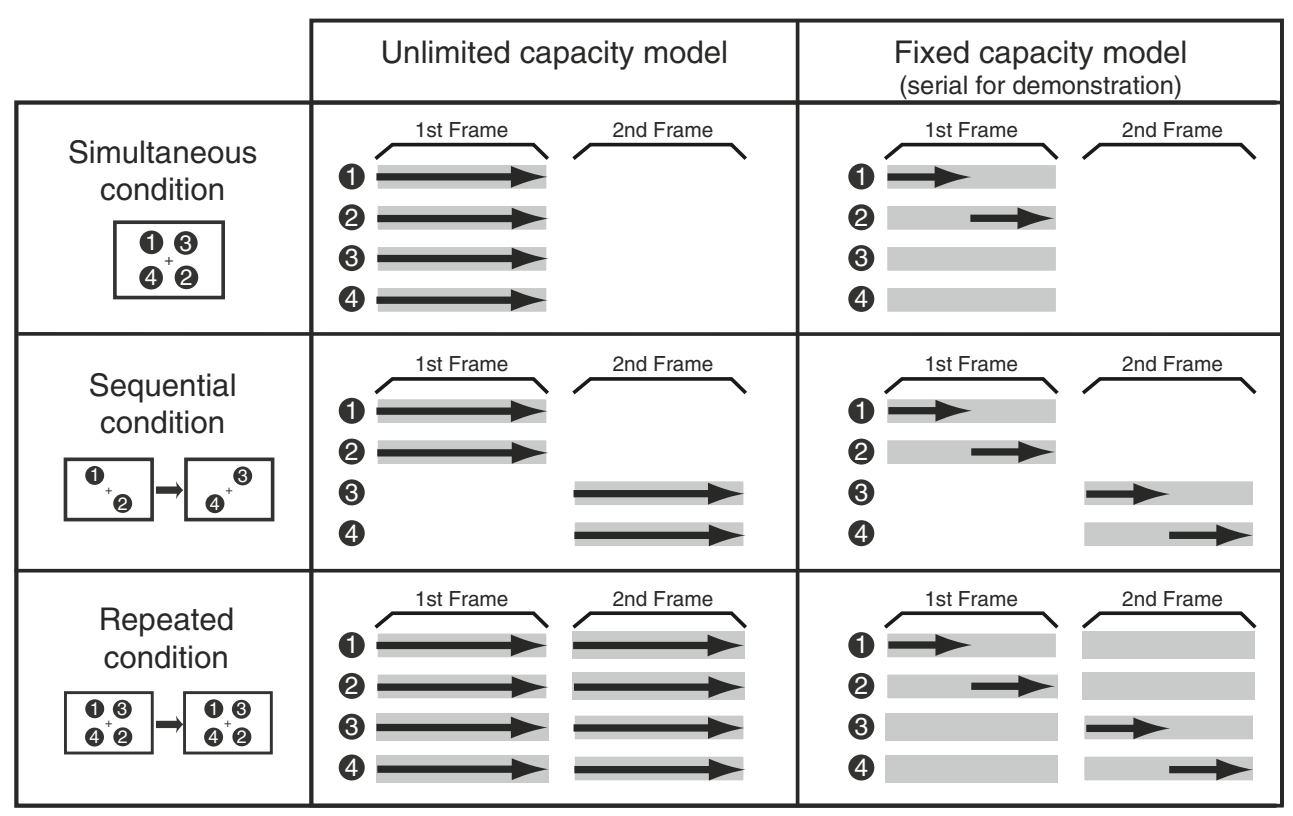

Fig. 2 Illustration of the predictions of unlimited-capacity and fixedcapacity models. In the unlimited-capacity model, performance is a function of exposure duration but not of the number of competing stimuli. Thus, the predicted pattern is simultaneous $=$ sequential $<$ repeated. In the fixed-capacity model, performance is a function of both exposure duration and competition among stimuli. The predic- tions of the model are demonstrated in terms of a serial model that allows two stimuli to be analyzed per frame. In the example shown, only two stimuli can be analyzed in the simultaneous condition, but four stimuli can be analyzed in both the sequential and repeated conditions. This yields the predicted pattern: simultaneous $<$ sequential $=$ repeated 
Figure 2 schematizes the logic for the predictions. In the figure, a gray bar indicates a stimulus display. The placement of each gray bar indicates how and when the stimulus appears on a trial: Locations are listed vertically and numbered 1 to 4 , whereas frames are listed horizontally and labeled "1st" or "2nd." The black arrows overlying the gray bars represent the observers' analysis of a stimulus.

Under the unlimited-capacity model, the observer analyzes all visible stimuli independently and in parallel. Accuracy is determined by the amount of time available to analyze the stimuli. In both the simultaneous and sequential conditions, each stimulus is displayed for $100 \mathrm{~ms}$, so the model predicts equivalent accuracy between these two conditions (Shiffrin \& Gardner, 1972). The repeated condition doubles the time available, and thus doubles the amount of information available to the observer, so better performance is expected in this condition.

To understand fixed capacity, consider a standard serial model in which the observer has time to process only two stimuli in each brief display (Fig. 2). In the simultaneous condition, the observer analyzes only two stimuli, leaving the observer no information about the others. In both the sequential and repeated conditions, the observer analyzes all four stimuli. Thus, overall performance is equivalent in the sequential and repeated conditions, and performance in the simultaneous condition is worse than in the other two. These predictions are general to many serial models, and to some fixed-capacity parallel models (Scharff et al., in press).

All predictions in this article assume that performance is limited by processing time. Thus, results from this paradigm can be interpreted only if performance in the repeated condition is better than performance in the simultaneous condition. This is not always true. In some studies, we have found cases with essentially identical performance in the simultaneous and repeated conditions. In these cases, performance might have been limited by internal noise that does not change with processing time. Adding the repeated condition provides an check of this critical assumption of the classic paradigm.

\section{Method}

The 6 observers were volunteers with normal or correctedto-normal visual acuity, and included author A.S. The apparatus was the same as in Scharff et al. (in press).

Stimuli Example stimuli are shown in Fig. 3. The stimulus set comprised 240 photographs of animals, divided into eight animal categories. Most images depicted a single animal, but 18 showed two or three animals (7.5\% of images). Each image was cropped to a square so that the animal(s) occupied most of the image. The images were rescaled to $100 \times 100$ pixels and converted to grayscale with 8-bit resolution. To equalize luminance and contrast, the pixel intensities were rescaled so that each image had a mean pixel intensity of 128 (of the possible 255) and a standard deviation of 43. As a result, the images all had the same mean luminance and an average pixel-by-pixel contrast of 0.33 . Masks were made by dividing each image into 100 squares $(10 \times 10)$ and randomly shuffling the squares.

Stimuli were presented at four corners of an imaginary square surrounding fixation. The stimuli subtended $3.9^{\circ}$ of visual angle and were centered at $3.0^{\circ}$ eccentricity. The centerto-center spacing of the neighboring stimuli was $4.2^{\circ}$, well exceeding the typical critical spacing for crowding effects (eccentricity $\times 1 / 2=1.5^{\circ}$ : Pelli \& Tillman, 2008).

Task Before each trial, the labels for two target categories were presented on screen-for example, "moose" and "squirrel." After the stimulus presentation, the two labels for the target categories reappeared, and the observer made an unspeeded response to indicate which one had appeared. The target was drawn from one of the target categories, and the distractors were randomly drawn from other categories.

\section{Results}

The results of Experiment 1 are plotted in Fig. 4. There was a reliable advantage for sequential over simultaneous presentation, with a mean within-observer difference of $10 \% \pm 1 \%$ (standard error of the mean difference) $[t(5)=17.55, p<$ $.0001]$. Between the sequential and repeated conditions, there was no reliable difference $(1 \% \pm 1 \%)[t(5)=1.32$, $p>.1]$. In the sequential condition, there was no effect of which frame included the target; the mean difference between first-frame-target and second-frame-target trials was $1 \% \pm 2 \%$ in favor of the second frame $[t(5)=0.43$, $p>.1]$. In sum, these results were consistent with a fixedcapacity model and rejected unlimited-capacity models.

\section{Experiment 2}

In Experiment 2, we evaluated whether the limits on performance found in Experiment 1 are a phenomenon of attention. Unambiguously attentional phenomena are under observer control, in contrast to sensory phenomena that are stimulus driven and not under observer control (for a similar experiment, see Palmer, 1994).

In Experiment 1, the simultaneous and sequential conditions used physically different displays: More images appeared per frame in the simultaneous condition. Thus, any performance difference might reflect sensory, as opposed to attentional, factors. For example, the observed capacity limitation might be explained by visual crowding (Pelli \& Tillman, 2008). 
Fig. 3 Example stimuli used in the study. Examples of all eight categories are shown. Each category included 30 images, of which 2 are shown

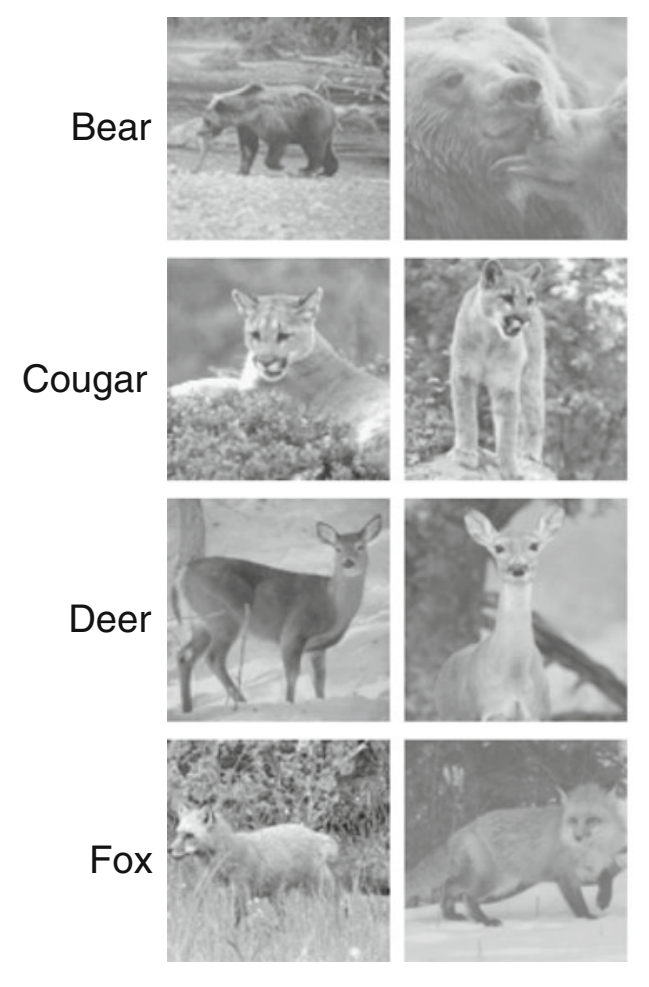

In Experiment 2, we eliminated sensory differences between conditions by using identical displays in each condition. Only the instructions varied between conditions. Because the conditions differed only in the instructions, any performance differences could not be attributed to sensory phenomena.

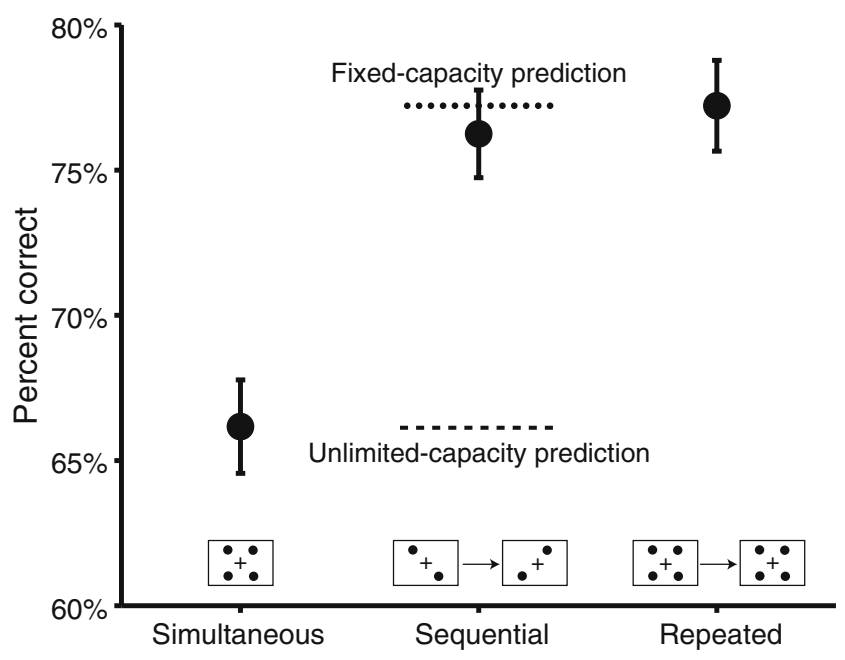

Fig. 4 Results of Experiment 1: Percent correct in the simultaneous, sequential, and repeated conditions, plotted with error bars representing \pm 1 standard error of the mean. The dotted and dashed lines represent predictions from the fixed-capacity models (sequential = repeated) and unlimited-capacity models (sequential = simultaneous), respectively

\section{Method}

Figure 5 illustrates the three conditions: neutral, cued simultaneous, and cued sequential. The second and third conditions correspond to the simultaneous and sequential conditions in Experiment 1. The neutral condition was added to check that the cues were effective. We did not include a repeated condition in Experiment 2 because it would require instructions and a stimulus presentation inconsistent with those for the other conditions. In the repeated condition of Experiment 1, an observer could rule out locations that had been analyzed in the first frame. This was not true for the cuing conditions used in Experiment 2.

The stimuli and display parameters were similar to those from Experiment 1. Each trial included eight unique stimuli (one target and seven distractors) displayed across two frames. Central precues indicated the relevant locations for each display frame. The cues were lines that pointed from the fixation point toward the relevant locations: They started $0.5^{\circ}$ away from fixation and ended $1^{\circ}$ away. Cues were displayed for $300 \mathrm{~ms}$ and were followed by a blank $300 \mathrm{~ms}$ interval before each stimulus display. The target always appeared in a cued location. The cues used in each condition are illustrated in Fig. 5. In the cued-sequential condition, cues indicated two stimuli from each frame. In the cued-simultaneous condition, cues indicated all four stimuli in one frame (either the first or the second) and no stimuli in the other frame. In the neutral condition, all 
Fig. 5 Schematic of the experimental conditions used in Experiment 2. Experiment 2 repeated the simultaneoussequential comparison using physically identical stimulus displays. Each condition included eight unique images, four in each frame, with only one target among them. Cues preceding each frame indicated possible target positions. The neutral condition indicates that the target can appear in any location in either frame. The simultaneous cues indicate that the target can appear in a particular frame (the second frame, in this example). The sequential cues indicate that the target can occur within a particular subset of each frame
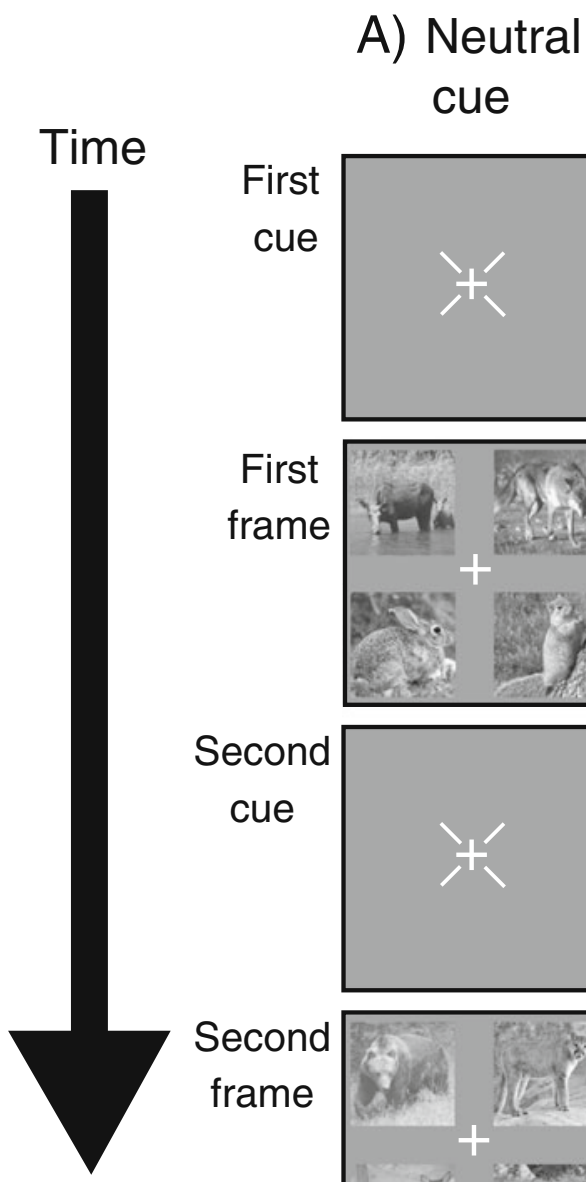

Second
cue
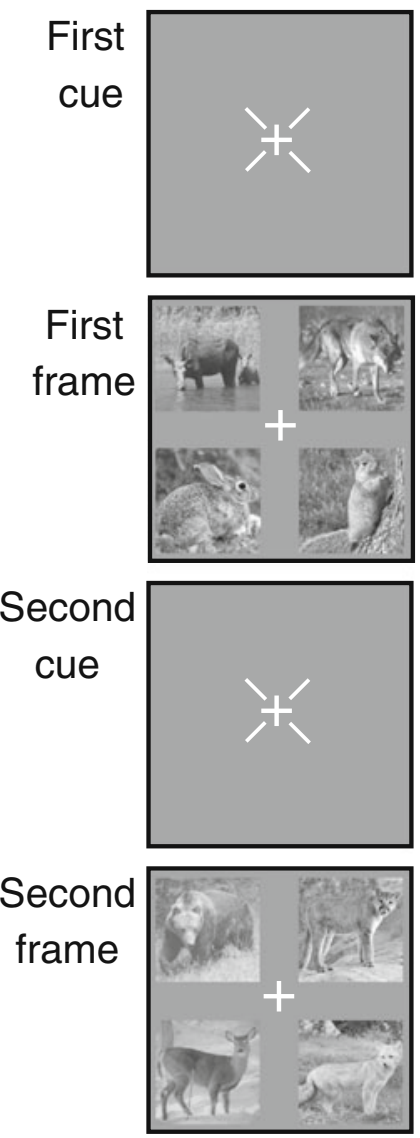


C) Cuedsequential
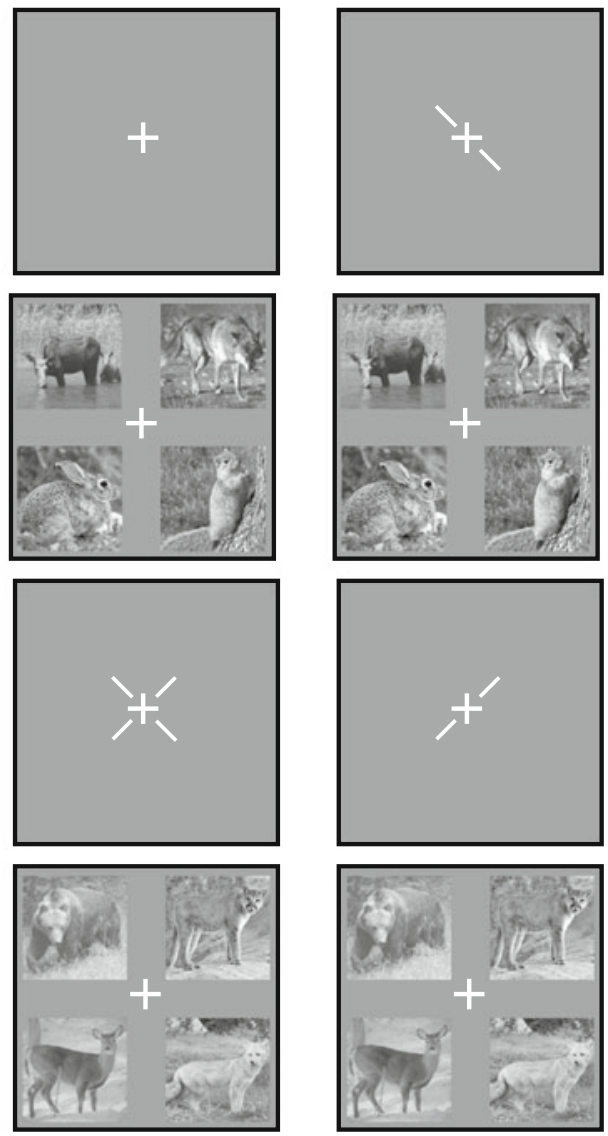

stimuli in both frames were cued. The conditions were run in separate blocks. A group of 5 observers completed Experiment 2, 4 of whom had completed Experiment 1. The new observer practiced Experiment 1 for several hours before beginning Experiment 2.

\section{Results}

The results of Experiment 2 are plotted in Fig. 6. There were reliable differences across cuing conditions, indicating that the cues were effective. There was a reliable advantage for the cued-sequential over the cued-simultaneous condition $(9 \% \pm 1 \%)[t(4)=6.18, p<.01]$. To check whether this effect was consistent with fixed capacity, we made two comparisons to the results from Experiment 1. First, we compared performance in the cued-sequential condition from Experiment 2 with performance in the repeated condition from Experiment 1. For the 4 observers who had participated in both experiments, there was no reliable difference between these conditions $(2 \% \pm 2 \%)[t(3)=$ $0.88, p>.4]$. Second, the magnitude of the simultaneoussequential effect in Experiment $2(9 \%)$ was similar to that



Fig. 6 Results of Experiment 2: Percent correct in the neutral, cuedsimultaneous, and cued-sequential conditions. The dashed line represents the prediction from unlimited-capacity models (cuedsequential $=$ cued-simultaneous). The dotted line represents the prediction from fixed-capacity models (cued-sequential $=$ repeated condition from Exp. 1). The neutral condition was included as a check for cue effectiveness; ineffective cuing would be indicated by equivalent performance in all three conditions 
in Experiment $1(10 \%)$. Both comparisons indicated that the effects in Experiment 2 were consistent with fixed capacity.

\section{Discussion}

Experiment 1 showed that the categorization of animal images had fixed capacity. Experiment 2 showed that the observed effect was attentional and not sensory. The results of Experiment 2 also indicated that the observer could control which objects were submitted to the fixed-capacity mechanism. Here, we will consider which stage or stages of processing limit the categorization of multiple objects.

Sensory, decision, and response stages

Several potential loci for the capacity limitation are ruled out by the logic of the present paradigm. Specifically, because there were four stimuli that had to be considered in all conditions, the simultaneous-sequential method prevented any set-size effects on decision and response. In addition, using identical stimuli in Experiment 2 ruled out sensory stages.

\section{Task difficulty}

One intuition is that difficult tasks have limited capacity, while easy tasks have unlimited capacity. Many manipulations influence task difficulty (e.g., stimulus contrast, stimulus duration), but whether a manipulation introduces or alleviates capacity limitations is a separate question. Moreover, several studies have indicated that capacity limitations do not depend on difficulty. Huang and Pashler (2005), for example, demonstrated a dissociation between difficulty and capacity using the simultaneous-sequential paradigm. They found that out of three difficult visual search tasks (feature, spatial conjunction, and feature conjunction), one showed evidence of being limited capacity (spatial conjunction), and the other two did not. For another example, Palmer (1994), Palmer et al. (2000), and Busey and Palmer (2008) manipulated both set size and the difficulty of a discrimination task and found that accuracy at all levels of difficulty was fit well by the same unlimited-capacity model. In sum, several studies have shown that capacity does not depend on task difficulty.

Stimulus eccentricity

A related explanation is that parafoveal presentation introduces capacity limits, because spatial resolution is limited in the visual periphery. This concern is partially addressed by the logic of the preceding paragraph: While stimulus eccentricity may affect task difficulty, there is no a priori reason to suspect that it introduces capacity limitations in divided attention. Furthermore, a major goal in the stimulus design was to make the stimuli as large and as close to fixation as possible without overlapping or invoking crowding. As a result, observers reported the subjective impression of clearly recognizing at least one or two of the images in each display. Consistent with this impression, the overall performance in the sequential condition was quite good, and presumably would improve further if the observers had needed to recognize only a single image presented at this eccentricity.

\section{Memory}

In principle, the sequential condition introduces an increased memory demand relative to the simultaneous condition. However, there were no reliable differences in performance for targets in the first versus the second frame of the sequential condition, indicating that memory demands did not influence performance over this time scale. Memory demands were otherwise consistent across conditions. Thus, the capacity limit cannot be due to memory.

\section{Perceptual learning}

To determine whether the degree of capacity limitation changed as the observers became more familiar with the task, we calculated a linear regression between the number of sessions completed and the simultaneous-minussequential difference for each session. The slope of this regression line was a $0.4 \% \pm 0.2 \%$ difference increment in percent correct per session $(r=.31, p>.5)$. This tiny effect, as compared to the overall $10 \%$ simultaneous-sequential difference, was in the wrong direction for learning to have accounted for the effects in these experiments.

Object perception and semantic categorization

With other explanations ruled out, we suggest that the fixed-capacity limit is due to either object perception or semantic categorization. The processing limit might be introduced during any of the perceptual processes that infer the physical form of objects, or by the semantic process of comparing perceptual objects with memory (Warrington \& Taylor, 1978). The object categorization task relies on both kinds of processing, so our experiments do not discriminate between these two explanations.

\section{Conclusion}

The present study adds to prior work showing that object categorization has limited capacity. Moreover, this study 
specifies the degree of the capacity limitation as fixed. This finding is consistent with object categorization by either the standard serial model or a fixed-capacity parallel model, such as the sample size model.

Author Note The authors are grateful to Chihiro Yonemori for help with collecting stimuli, and to Todd Horowitz, Liqiang Huang, Bill Prinzmetal, and an anonymous reviewer for their helpful comments on an earlier draft of this article. This research was supported by a grant from the University of Washington's Royalty Research Fund.

\section{References}

Biederman, I., Blickle, T. W., Teitelbaum, R. C., \& Klatsky, G. J. (1988). Object search in nonscene displays. Journal of Experimental Psychology. Learning, Memory, and Cognition, 14, 456467. doi:10.1037/0278-7393.14.3.456

Bonnel, A. M., Stein, J. F., \& Bertucci, P. (1992). Does attention modulate the perception of luminance changes? The Quarterly Journal of Experimental Psychology, 44A, 601-626.

Broadbent, D. E. (1958). Perception and communication. New York: Pergamon Press.

Busey, T., \& Palmer, J. (2008). Set-size effects for identification versus localization depend on the visual search task. Journal of Experimental Psychology. Human Perception and Performance, 34, 790-810.

Davis, E. T., Shikano, T., Peterson, S. A., \& Michel, R. K. (2003). Divided attention and visual search for simple versus complex features. Vision Research, 43, 2213-2232.

Duncan, J., Ward, R., \& Shapiro, K. (1994). Direct measurement of attentional dwell time in human vision. Nature, 369, 313-315.

Evans, K. K., \& Treisman, A. (2005). Perception of objects in natural scenes: Is it really attention free? Journal of Experimental Psychology. Human Perception and Performance, 31, 1476-1492.

Gardner, G. T. (1973). Evidence for independent parallel channels in tachistoscopic perception. Cognitive Psychology, 4, 130-155.

Huang, L., \& Pashler, H. (2005). Attention capacity and task difficulty in visual search. Cognition, 94, 101-111.

Kahneman, D., Treisman, A., \& Gibbs, B. J. (1992). The reviewing of object files: Object-specific integration of information. Cognitive Psychology, 24, 175-219.

Mozer, M. (1991). The perception of multiple objects. Cambridge: MIT Press.

Neisser, U. (1967). Cognitive psychology. New York: Meredith.
Norman, D. A., \& Bobrow, D. G. (1975). On data-limited and resource-limited processes. Cognitive Psychology, 7, 44-64.

Palmer, J. (1994). Set-size effects in visual search: The effect of attention is independent of the stimulus for simple tasks. Vision Research, 34, 1703-1721.

Palmer, J. (1995). Attention in visual search: Distinguishing four causes of a set-size effect. Current Directions in Psychological Science, 4, 118-123.

Palmer, J., Verghese, P., \& Pavel, M. (2000). The psychophysics of visual search. Vision Research, 40, 1227-1268.

Pelli, D. G., \& Tillman, K. A. (2008). The uncrowded window of object recognition. Nature Neuroscience, 11, 1129-1135.

Potter, M. C., \& Fox, L. F. (2009). Detecting and remembering simultaneous pictures in a rapid serial visual presentation. Journal of Experimental Psychology. Human Perception and Performance, 35, 28-38.

Rousselet, G. A., Fabre-Thorpe, M., \& Thorpe, S. J. (2002). Parallel processing in high-level categorization of natural images. Nature Neuroscience, 5, 629-630.

Rousselet, G. A., Thorpe, S. J., \& Fabre-Thorpe, M. (2004a). How parallel is visual processing in the ventral pathway? Trends in Cognitive Sciences, 8, 363-370.

Rousselet, G. A., Thorpe, S. J., \& Fabre-Thorpe, M. (2004b). Processing of one, two or four natural scenes in humans: The limits of parallelism. Vision Research, 44, 877-894.

Scharff, A., Palmer, J., \& Moore, C. M. (2011). Extending the simultaneous-sequential paradigm to measure perceptual capacity for features and words. Journal of Experimental Psychology: Human Perception and Performance. doi:10.1037/a0021440

Shaw, M. L. (1980). Identifying attentional and decision-making components in information processing. Attention and performance VIII (pp. 277-296). Hillsdale: Erlbaum.

Shiffrin, R. M., \& Gardner, G. T. (1972). Visual processing capacity and attentional control. Journal of Experimental Psychology, 93, $72-83$.

Shiffrin, R. M., \& Schneider, W. (1977). Controlled and automatic human information processing: II. Perceptual learning, automatic attending, and a general theory. Psychological Review, 84, 127190.

Starr, M., \& Rayner, K. (2001). Eye movements during reading: Some current controversies. Trends in Cognitive Sciences, 5, 156-163.

Townsend, J. T. (1974). Issues and models concerning the processing of a finite number of inputs. In B. H. Kantowitz (Ed.), Human information processing: Tutorials in performance and cognition (pp. 133-185). Hillsdale: Erlbaum.

Warrington, E. K., \& Taylor, A. M. (1978). Two categorical stages of object recognition. Perception, 7, 695-705. doi:10.1068/ p070695 